\title{
New therapeutic agents in diabetic nephropathy
}

\author{
Yaeni Kim and Cheol Whee Park
}

Division of Nephrology, Department of Internal Medicine, College of Medicine, The Catholic University of Korea, Seoul, Korea

Received: May 19, 2016 Accepted: December 25, 2016

\section{Correspondence to}

Cheol Whee Park, M.D.

Division of Nephrology,

Department of Internal

Medicine, College of Medicine,

Seoul St. Mary's Hospital, The

Catholic University of Korea, 222

Banpo-daero, Seocho-gu, Seoul

06591, Korea

Tel: $+82-2-2258-6038$

Fax: +82-2-599-3589

E-mail: cheolwhee@hanmail.net
Studies investigating diabetic nephropathy (DN) have mostly focused on interpreting the pathologic molecular mechanisms of $\mathrm{DN}$, which may provide valuable tools for early diagnosis and prevention of disease onset and progression. Currently, there are few therapeutic drugs for DN, which mainly consist of antihypertensive and antiproteinuric measures that arise from strict renin-angiotensin-aldosterone system inactivation. However, these traditional therapies are suboptimal and there is a clear, unmet need for treatments that offer effective schemes beyond glucose control. The complexity and heterogeneity of the DN entity, along with ambiguous renal endpoints that may deter accurate appraisal of new drug potency, contribute to a worsening of the situation. To address these issues, current research into original therapies to treat $\mathrm{DN}$ is focusing on the intrinsic renal pathways that intervene with intracellular signaling of anti-inflammatory, antifibrotic, and metabolic pathways. Mounting evidence in support of the favorable metabolic effects of these novel agents with respect to the renal aspects of DN supports the likelihood of systemic beneficial effects as well. Thus, when translated into clinical use, these novel agents would also address the comorbid factors associated with diabetes, such as obesity and risk of cardiovascular disease. This review will provide a discussion of the promising and effective therapeutic agents for the management of DN.

Keywords: AMP-activated protein kinases; Diabetic nephropathies; Incretins; Sodium/glucose co-transporter 2

\section{INTRODUCTION}

Chronic kidney disease (CKD) has become a worldwide health-care concern that calls for prompt initiatives and firm commitments to find effective treatments. The prevalence of CKD is estimated to be $8 \%$ to $16 \%$ globally and is steadily rising [1]. It not only causes pain in individuals with the disease, but also has effects on society. CKD patients are exposed to an increased risk of death, comorbidities, and cognitive impairment, which all contribute to poor quality of life. Moreover, the medical expenses covering hospitalizations for both cardiovascular and non-cardiovascular events and all-cause mortality are costly. Unfortunately, the end-stage renal disease
(ESRD) population is expanding and the CKD population contains potential ESRD patients. Consequently, the expected number of individuals requiring renal replacement therapy (RRT) is tremendous. An example of this prognosis is as follows: if there are currently 100 patients with stage 3 CKD with estimated glomerular filtration rates (eGFRs) lower than $60 \mathrm{~mL} / \mathrm{min} / 1.73 \mathrm{~m}^{2}$ 10 years from now, 65 of these 100 patients would have already died due to cardiovascular complications, eight would be in need of RRT, and 27 would have ongoing CKD [2]. As the CKD stage deteriorates, there is a consistent increase in the rates of RRT requirement and death. A far greater effect than a doubling of the rates of death and RRT was observed in a 5-year longitudinal fol- 
low-up of 27,998 patients, with a mortality and RRT rate of $19.5 \%$ and $1.1 \%$, respectively, in patients with stage 2 CKD, and of $45.7 \%$ and $19.9 \%$, respectively, in patients with stage 4 CKD [3]. The substantial number of individuals experiencing premature death highlights the urgent need for intervention.

The rise in the prevalence of CKD, along with the subsequent increase in mortality rate, is in part due to the increasing population of the elderly and individuals with diabetes, metabolic syndrome, and cardiovascular disease [4]. As a cause of ESRD, diabetic nephropathy (DN) ranks first both globally and nationwide, with its incidence exponentially increasing from $10 \%$ in 1985 to $48 \%$ in 2014 according to the Korean ESRD registry $[5,6]$. DN is clinically characterized by proteinuria (mostly albuminuria), elevated serum creatinine levels, and decreased eGFR. It is well recognized that tight glucose and blood pressure control with renin-angiotensin-aldosterone system (RAAS) blockade constitute the backbone of management strategies for DN. In fact, RAAS blockade has proven to be effective in reducing albuminuria and alleviating the deterioration of DN. Nevertheless, an ever-increasing portion of the DN population is underserved; therefore, seeks novel treatment alternatives. In hopes that treating and deterring the progression of DN may help the prospects of patients with this disease, this review presents recent discoveries and attempts to address some of the unresolved questions that warrant contemplation to provide future direction to overcoming this disease entity.

\section{PATHOGENESIS OF DIABETIC NEPHROPATHY}

Hemodynamic and metabolic factors, among which chronic hyperglycemia and dyslipidemia are assumed to play pivotal roles, interact to contribute to the development of DN [7]. The early asymptomatic phase of DN is characterized by an increased glomerular filtration rate (GFR) and renal plasma flow [8]. During hyperglycemia, increased amounts of intraluminal glucose and sodium in the proximal tubule are reabsorbed by upregulation and activation of various co-transporters and exchangers in an effort to achieve euglycemia. Glomerular hyperfiltration results from this hyper-reabsorption in the proximal tubule, which then reduces salt delivery to the macula densa and further decreases blood flow resistance in the afferent arteriole of the glomerulus, contributing to a net increase in single-nephron GFR $[9,10]$. Hyperglycemia also contributes to the well-recognized pathologic molecular pathways that contribute to renal injury. The production of advanced glycation end products activates polyol and the hexosamine pathways to stimulate the formation of protein kinase C. Mitochondrial overproduction of superoxide exacerbates oxidative stress and promotes inflammation and fibrosis, which induce both functional and structural injuries to the kidney $[11,12]$. The morphological changes associated with early phase DN comprise thickening of the glomerular basement membrane (GBM), tubular basement membrane and mesangial expansion with characteristic Kimmelstiel-Wilson nodules, along with tubulointerstitial changes and hyalinosis, which result in the replacement of arteriolar smooth muscle cells and the development of sclerotic glomeruli [13].

Dyslipidemia in diabetic patients, especially in type 2 diabetes, is a major reversible risk factor for the progression of renal disease and cardiovascular mortality $[14,15]$. Interestingly, sustained hyperglycemia in diabetes increases free fatty acid (FFA) synthesis and triglyceride (TG) accumulation in adipose tissue. Further elevation of serum TGs, FFAs, and modified cholesterol levels causes ectopic accumulation of lipids in the parenchymal organs including the pancreas, liver, heart, and kidneys. This process of lipotoxicity serves as an aggravating factor in the pathogenesis of $\mathrm{DN}$ in association with glomerulosclerosis and tubulointerstitial injury $[16,17]$. This lipid byproduct accumulation in DN may arise from altered lipid metabolism resulting from a mismatch between lipid uptake and disposal, featuring enhanced lipid uptake and reduced peroxidation, catabolism, and efflux of residues in the kidney [18].

Systemic hypertension could be both the cause of renal functional deterioration and the consequence of renal damage. Systemically elevated blood pressure due to the co-existence of obesity and cardiovascular disease causes increases in intraglomerular pressure and hyperfiltration, along with subsequent proteinuria [19].

Proteinuria contributes to the development of glomerular hypertrophy and sclerosis by producing local proinflammatory and prosclerotic effects. Both tubular and tubulointerstitial alterations, including hypertro- 
phy, fibrosis, and eventual tubular atrophy, occur along with glomerular changes [13]. In accordance with this, disruption of renal vascular autoregulation by overproduction of vasoactive factors further exacerbates glomerular hyperfiltration. RAAS activation plays a central role by contributing to increased glomerular capillary hydraulic pressure [8]. The production of local angiotensin II increases intraglomerular pressure and proteinuria and stimulates local release of cytokines, further activating inflammatory pathways. This vicious cycle of glomerular hypertrophy and sclerosis, tubulointerstitial inflammation, and fibrosis independently and collectively causes and perpetuates the exacerbation of renal damage $[20,21]$. Persistent proteinuria followed by a progressive decline in eGFR is characteristic of the late clinical phase of $\mathrm{DN}$, with negatively charged proteoglycan loss in the GBM, podocyte injuries, and malfunctioning tubular cells that ultimately lead to $\operatorname{ESRD}[13,22]$. Furthermore, other factors that potentially contribute to the progression of $\mathrm{DN}$ include repetitive episodes of acute kidney injury, comorbid renal and vascular diseases, hyperuricemia, systemic and local inflammation, and tubular cell injury resulting from high glucose exposure.

\section{RENOPROTECTIVE STRATEGIES FOR DIABETIC NEPHROPATHY: THE TRIUMVIRATE}

Based on the results of large randomized clinical trials (RCTs), intensive control of glucose and blood pressure might delay the onset, and deter the progression, of DN. Along with this, RAAS blockade constitutes an essential component of the triumviral management strategy for DN. Strict control of both glucose and blood pressure levels can largely influence the rate of eGFR decline. Indeed, albuminuria in patients with $\mathrm{DN}$ is associated with a linear decline in GFR, progression to ESRD, and cardiovascular mortality; thus, any means of reducing the extent of proteinuria would be a promising treatment in preventing the progression of DN [23-25].

Deciding whether glycemic control or blood pressure control is of more importance in preventing the progression of DN depends on the clinical circumstances of the individual. During the progression of normoalbuminuria to microalbuminuria, glycemic control plays an im- portant role; blood pressure control; however, does not seem to exert a prominent effect on disease progression. Once microalbuminuria has shifted into a macroalbuminuric state, blood pressure control is of greater importance than glycemic control, in that strict blood pressure restraint helps prevent exacerbation of persistent proteinuria along with renal functional deterioration. However, the significance of glucose control spans the entire disease course, as uncontrolled blood glucose is highly associated with cardiovascular events at any stage of DN. Glycosylated hemoglobin (HbAlc) targets of 6.5\% versus $7 \%$ and blood pressure targets of less than $125 / 75$ $\mathrm{mmHg}$ versus $130 / 80 \mathrm{mmHg}$ versus $140 / 80 \mathrm{mmHg}$ are constantly disputed. The Steno-2 study confirmed that a target-driven, long-term, intensified intervention aimed at multiple risk factors (blood pressure $<130 / 80 \mathrm{mmHg}$, proteinuria < $0.3 \mathrm{~g} /$ day, low density lipoprotein [LDL] $<100 \mathrm{mg} / \mathrm{dL}, \mathrm{LDL}+$ very low density lipoprotein $<130$ $\mathrm{mg} / \mathrm{dL}, \mathrm{HbAlc}<7.0 \%$, smoking cessation, and near normalization of anemia of hemoglobin of 11 to $13 \mathrm{~g} / \mathrm{dL}$ ) in type 2 diabetes patients with microalbuminuria significantly reduced the risks of cardiovascular and microvascular events, including nephropathy, retinopathy, and autonomic neuropathy by approximately 50\% [26]. However, there is a growing body of counter-evidence showing that stricter established target levels result in a greater chance of hypoglycemia and cerebrovascular ischemic events in the elderly. Thus, the treatment targets should be tailored according to each individual's clinical situation to optimize renal outcome.

In terms of RAAS inhibition, there have been various attempts to optimize RAAS inactivation with the least number of adverse effects. Several trials have been conducted to validate the efficacy of high dose angiotensin converting enzyme inhibitors (ACEI) or angiotensin II type 1 receptor blockers (ARBs) alone, the combined use of ACEI and ARBs, renin inhibitors (aliskiren), aldosterone inhibitors (spironolactone or eplerenone), and angiotensin II type 2 receptor enhancement. The following studies have reported some promising results. Both the Reduction of Endpoints in NIDDM with the Angiotensin II Antagonist Losartan (RENAAL) trial and the Irbesartan Diabetic Nephropathy Trial (IDNT) showed that ARBs slowed the progression of type $2 \mathrm{DN}$ by slowing the rate of GFR decline compared to placebo groups. This use of ACEIs and ARBs may only provide 
suboptimal RAAS inhibition and there may still be considerable room for improvement since the normal rate of GFR decline due to aging in those without DN is only $1 \mathrm{~mL} / \mathrm{min} / 1.73 \mathrm{~m}^{2} /$ year $[27,28]$. Moreover, a compensatory increase in plasma renin activity as a result of angiotensin blockade led to alterations in angiotensin production and conversion, which suggest the need for direct renin inhibition. Aliskiren is a once-daily, orally effective, small-molecule renin inhibitor that directly targets angiotensin production at its rate-limiting step. It has been proven to produce a non-inferior blood pressure lowering effect relative to that of ARBs and ACEIs with an insignificant side effect profile. "ACE escape," a phenomenon associated with uncontrolled blood pressure and heart failure, can be avoided with the use of a direct renin inhibitor, which may otherwise occur due to the increased production of angiotensin II with a reactive increase in plasma renin activity through ACE-independent pathways [29-31].

Moreover, aliskiren treatment with losartan proved effective in that it significantly reduced albuminuria and the rate of decline in eGFR, except for relatively infrequent occurrences of hyperkalemia, independently of the baseline CKD stage in patients with DN [32]. A meta-analysis of 11 trials involving 991 patients showed that use of a nonselective aldosterone antagonist along with ACEIs and/or ARBs significantly reduced 24-hour proteinuria and blood pressure in comparison with ACEIs and/or ARBs plus placebo. However, this reduction in albuminuria did not translate into an improvement in GFR and there was a significant increase in the risk of hyperkalemia with the addition of a nonselective aldosterone antagonist to ACEIs and/or ARBs. The longterm effects of these agents on renal outcome, mortality, and safety need to be established [33]. As a last resort for the optimization of RAAS inactivation, dual therapies involving either ACEIs plus ARBs or renin inhibitors plus aldosterone inhibitors were evaluated. Despite these efforts, insignificant effects on renal function as well as increased risks of hyperkalemia and acute kidney injury were demonstrated by both the ALTITUDE (RAS inhibition plus aliskiren) and the VA NEPHRON-D (losartan plus lisinopril) trials.

The effect of statins compared with placebo or no treatment was evaluated in 80 trials with 51,099 individuals. While statins significantly lowered mortality and cardiovascular events in individuals within the early stages of CKD, they had little or no effects in those on dialysis, and had uncertain effects in kidney transplant recipients [34]. Although the mechanism through which statins exert their effects on stroke and kidney function remain to be elucidated, it is certain that statins play an important role in the primary prevention of cardiovascular events and mortality in CKD patients [35].

\section{EMERGING NEW AGENTS IN DIABETIC NE- PHROPATHY: BEYOND RAAS INHIBITION}

Clinical trials with renal endpoints in patients with diabetes mellitus aim to provide add-on therapies to RAAS blockade. These newer pharmaceuticals are delicately designed to activate or inactivate potentially engaged pathways in the disease course and the narrower spectrums of the drugs are designed to amplify the target effect (Table 1).

Some of these newer drugs for which terminated RCTs have shown negative results include bardoxolone methyl, avosentan, pirfenidone, anti-connective tissue growth factor antibody FG3019, sulodexide, N-acetylcysteine, and ruboxistaurin. Specifically, the Avosentan on Time to Doubling of Serum Creatinine, End Stage Renal Disease or Death in Patients With Type 2 Diabetes Mellitus and Diabetic Nephropathy (ASCEND) trial, which investigated the endothelin receptor antagonist avosentan, was terminated due to an increased risk of fluid overload and consequent congestive heart failure resulting from proximal tubular sodium reabsorption, despite its favorable effect of reducing albuminuria when added to standard treatment [36]. Moreover, dose-dependent peripheral edema is a major barrier limiting their routine use in DN patients. The Ongoing Study of Diabetic Nephropathy With Atrasentan (SONAR) phase III trial with an endothelin A receptor antagonist is approaching its closing date, though some safety issues concerning edema and heart disease are constantly being raised [37]. Bardoxolone, once expected to produce dramatic and powerful antioxidative and anti-inflammatory effects through the activation of the nuclear 1 factor-related factor 2 transcription factor, failed to produce promising results. It was dropped from the Bardoxolone Methyl Treatment: Renal Function in CKD/Type 2 
Table 1. New therapeutic agents in diabetic nephropathy beyond conventional RAAS inhibition

\begin{tabular}{l}
\hline Glucose-dependent pathway \\
AGEs reduction: aminoguanidine, pyridoxamine \\
PPAR agonists: fenofibrate, thiazolidinediones \\
Incretins: GLP-1 receptor, DPP-4 inhibitor \\
SGLT2 inhibitor: canagliflozin, dapagliflozin \\
AMPK activator: AICAR, adiponectin, statins \\
Vasoactive-pathway \\
RAAS system: aliskiren, HRP, spironolactone, eplerenone \\
Endothelial antagonism: avosentan, atrasentan \\
Intracellular signaling \\
PKC- $\beta$ inhibition: ruboxistaurin \\
Rho kinase inhibitors: fasudil \\
Prosclerotic GFs inhibition \\
Anti-PDGF, anti-TGF- $\beta$, anti-CTGF, pirfenidone \\
Anti-inflammatory pathway \\
Adenosine, pentoxifylline (TNF- $\alpha$ inhibition), \\
adiponectin, statins, mTOR inhibitor \\
Anti-oxidative pathway \\
Bardoxolone methyl, N-acetylcysteine, probucol \\
Others \\
Glycosaminoglycan, sulodexide, paricalcitol
\end{tabular}

RAAS, renin-angiotensin-aldosterone system; AGE, advanced glycation end product; PPAR, peroxisome proliferator-activated receptor; GLP-1, glucagon-like peptide 1; DPP-4, dipeptidyl peptidase 4; SGLT2, sodium/glucose cotransporter 2; AMPK, adenosine monophosphate-activated protein kinase; AICAR, 5-aminoimidazole-4-carboxamide ribonucleoside; HRP, hand-region peptide; $\mathrm{PKC}-\beta$, protein kinase $\mathrm{C} \beta$; GF, growth factor; PDGF, platelet-derived growth factor; TGF- $\beta$, transforming growth factor $\beta$; CTGF, connective tissue growth factor; TNF- $\alpha$, tumor necrosis factor $\alpha$; mTOR, mammalian target of rapamycin.

Diabetes (BEAM) and Bardoxolone Methyl Evaluation in Patients with Chronic Kidney Disease and Type 2 Diabetes: the Occurrence of Renal Events (BEACON) trials, owing to the development of various degrees of side effects ranging from trivial muscle cramps to serious issues including heart failure, non-fatal myocardial infarction, stroke, and cardiovascular mortality. It also increased urine albumin excretion and blood pressure [38]. Sulodexide, a highly purified mixture of glycosaminoglycans that potentiates the antiprotease activities of both antithrombin III and heparin cofactor II, had shown beneficial effects in diabetic animal models but failed to prove efficacious in the sulodexide macroalbuminuria (Sun-MACRO) trial [39]. Results from some on- going RCTs with unestablished renal end points are as follows. Paricalcitol seems effective only at a high dose $(2 \mu \mathrm{g})$, and showed only proteinuria lowering effects in the selective vitamin $\mathrm{D}$ receptor activation with paricalcitol for reduction of albuminuria in patients with type 2 diabetes (VITAL) study. Evidence regarding its effect in slowing the progression of CKD has yet to be demonstrated [40]. The Proteomic prediction and Renin angiotensin aldosterone system Inhibition prevention Of early diabetic nephRopathy in TYpe 2 diabetic patients with normoalbuminuria (PRIORITY) trial using mineralocorticoid receptor blockers (MRAs; MT-3995, BAY 94-3995, and BAY 94-8862) has shown limited efficacy in the early stages of DN and concerns regarding the development of hyperkalemia need to be addressed [41]. The efficacy of xanthine oxidase inhibitors in terms of delaying the decline in the rate of eGFR was proven in a relatively small sample of CKD patients and will need to be validated in a larger population [42]. The favorable effect of an anti-transforming growth factor $\beta$ (TGF- $\beta$ ) antibody (LY2382770), which was demonstrated in an animal model, has not yet been translated into human data. Pentoxifylline is a methylxanthine derivative and nonspecific phosphodiesterase inhibitor that has anti-inflammatory, antiproliferative, and ant-fibrotic actions. At the end of the Pentoxifylline for Renoprotection in Diabetic Nephropathy (PREDIAN) trial, the rate of eGFR decline and the change in urinary albumin excretion were significantly lower in study participants taking the drug as compared to the placebo group [43]. Stem cell therapy in experimental DN models has not yet shown efficacy in either diabetic mice or rat models $[44,45]$.

The failure rate of new agents investigated in RCTs exceeds $90 \%$, with barely half of the agents entering phase III trials. These disappointing results may be due to the heterogeneity of the disease entity with its various causes and its diverse underlying pathogenic mechanisms that may be intertwined. Moreover, the flexible inclusion criteria of the trials, including variable stages of DN ranging from relatively spared renal function to advanced CKD, may make it difficult to infer causality. Although changes in proteinuria levels are frequently used as a parameter in predicting renal outcome, proteinuria per se cannot be translated into eGFR; thus, other consolidated surrogate endpoints representing renal 

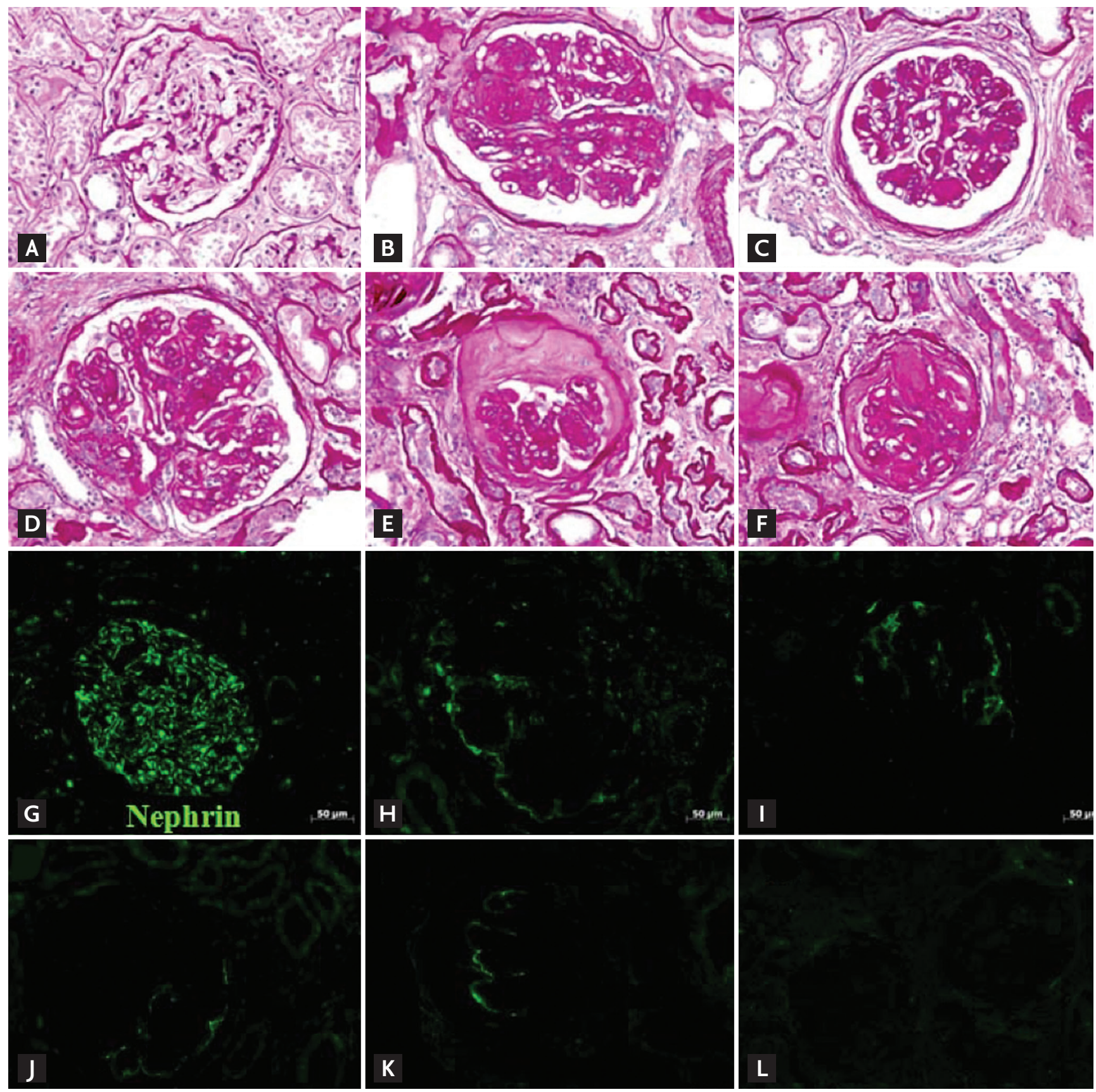

Figure 1. Changes in glomerular phenotypes (A-F, PAS stain) and nephrin (G-L, immunofluorescence analysis of nephrin in green) expressions of human diabetic kidneys according to the stages of chronic kidney disease (CKD). The stage of the disease is assigned based on the level of kidney function as defined by estimated glomerular filtration rates (eGFRs) (×400): (A, G) normal healthy control; $(\mathrm{B}, \mathrm{H})$ stage 1 with urine protein to creatinine ratio (P/C) of $4.49 \mathrm{mg} / \mathrm{dL}$; (C, I) stage 2 with P/C $4.64 \mathrm{mg} / \mathrm{dL}$; (D, J) stage 3 with P/C $10.4 \mathrm{mg} / \mathrm{dL}$; (E, K) stage 4 with P/C $4.7 \mathrm{mg} / \mathrm{dL}$; and (F, L) stage 5 with P/C $11.3 \mathrm{mg} / \mathrm{dL}$. Irrespective of the CKD stage in type 2 diabetic nephropathy, (B-F) the glomerular extracellular matrix expansion and sclerosis are prominent and (HL) glomerular nephrin expressions are scarce throughout the whole stages (A, G) when compared to that of normal glomeruli. (L) It is noteworthy to point out complete obliteration of glomerular nephrin expressions in CKD stage 5. These findings suggest that albuminuria and eGFR do not serve as reliable variables to reflect the true renal phenotype of each individual with different renal functions. 
outcomes are needed (Fig. 1). Despite the inconsistent and questionable results of the trials involving newly developed agents, the following agents have been proven to demonstrate somewhat favorable renoprotective effects through inhibiting intrinsic renal pathways linked to inflammation and fibrosis.

\section{Incretin-based agents}

Incretins are gut hormones that are secreted into the circulation postprandially to exert insulinotropic activity. Glucose-dependent insulinotropic polypeptide (GIP) and glucagon-like peptide 1 (GLP-1) are the most important incretins released in humans, as their potential use for the treatment of diabetes appears promising. Impaired incretin regulation is implied in type 2 diabetes based on findings that meal-induced increases in GLP-1 levels were reduced, whereas the level of GIP was retained in the normal range in subjects with diabetes [46]. Thus, defective GLP-1 release may be responsible for the characteristic metabolic disarray presented in type 2 diabetes. Although the mechanism involved in defective GLP-1 secretion has been unraveled, the preservation of the GLP-1 response to oral glucose in those with prediabetes suggests that its impaired release may be a consequence of the diabetic condition rather than the primary incident [47]. Moreover, the insulin response to exogenously administered GIP is reduced in type 2 diabetes [48]. This impaired secretion of GLP-1 and the defective insulinotropic action of GIP constitute potential therapeutic targets in subjects with type 2 diabetes. The potential use of these insulinotropic gut peptides has been most successful for GLP-1, which exerts antidiabetogenic properties by stimulating insulin secretion, increasing $\beta$-cell mass, inhibiting glucagon secretion, delaying gastric emptying, and inducing satiety [49-51]. Despite these favorable results with GLP-1, its rapid degradation by dipeptidyl peptidase 4 (DPP-4) makes it somewhat impractical. However, this limitation can be overcome using DPP-4 resistant GLP-1 receptor agonists and DPP- 4 inhibitors. Apart from their renowned glycemic effects of improving insulin secretion and inhibiting glucagon secretion, both GLP-1 receptor agonists and DPP-4 inhibitors are known to reduce blood pressure, dyslipidemia, and inflammation to a certain degree with low risk of hypoglycemia (Fig. 2).

With respect to their renal effects, these incretin-based agents have been shown to reduce albuminuria by inhibiting renal tubular sodium reabsorption and subsequent increases in glomerular pressure in both rodents and humans. Furthermore, the onset of the morphological alterations associated with DN were delayed or prevented with these drugs in rodent models [52]. There are currently a few results from phase III trials suggesting the potential therapeutic effects of sitagliptin, alogliptin, and linagliptin in significantly reducing the amount of albuminuria in patients with type 2 diabetes mellitus [53-55]. Treatment with exendin-4 or exenatide has been shown to be associated with reductions in both albuminuria and urinary levels of TGF- $\beta$ and type IV collagen in the same population [56]. Although it is well recognized that renal fibrosis is primarily mediated through TGF- $\beta$, there is limited clinical evidence of the effect of targeting TGF- $\beta$. The anti-fibrotic effect of linagliptin was demonstrated in a type 1 model of DN after 4 weeks of treatment. The mitigation of fibrotic changes due to DN was associated with an inhibition of endo-

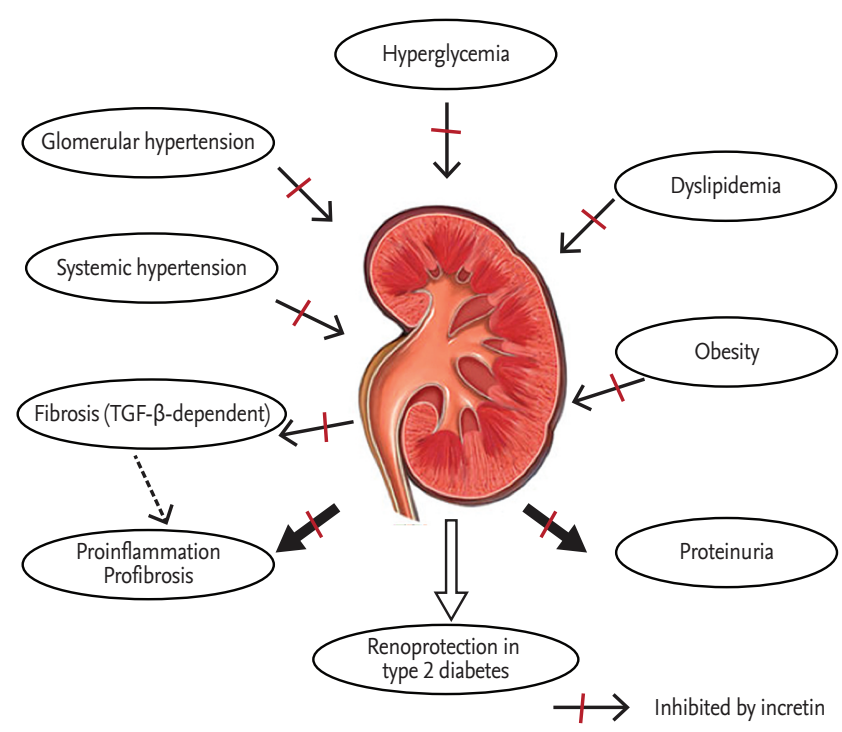

Figure 2. Favorable renal outcome achieved by incretin in diabetic nephropathy. Glucagon-like peptide 1 receptor agonists mimic favorable actions of incretin. Its glycemic effect via improving insulin secretion and inhibiting glucagon secretion, antihypertensive effect in both systemic and glomerular hypertension with attenuated dyslipidemia and obesity collectively ameliorate proteinuria and blocks proinflammatory and profibrotic pathways through transforming growth factor $\beta$ (TGF- $\beta$ )-dependent anti-fibrotic effect. These results comprehensively contribute to the renoprotection in type 2 diabetes. 
thelial-to-mesenchymal transition and the restoration of microRNA 29s [57]. In human renal proximal tubular cells, linagliptin seems to interfere with the activation of TGF- $\beta$. Moreover, the cation-independent mannose 6-phosphate receptor (CIM6PR) is crucial for the conversion of latent to active TGF- $\beta 1$ in human kidney proximal tubular cells [58]. The co-existence of DPP-4 and CIM6PR on the cell membrane is highly suggestive of the reduced activation of TGF- $\beta$, through which linagliptin may have exerted its TGF- $\beta 1$-dependent antifibrotic effects (Fig. 2) [59]. From a clinical standpoint, this finding is relevant because of the potential of linagliptin as an antifibrotic agent in patients with diabetes.

\section{Sodium/glucose co-transporter $\mathbf{2}$ inhibitors}

Sodium/glucose co-transporter 2 (SGLT2) inhibitors are promising hypoglycemic agents that have the added advantage of not promoting hyperinsulinemia, weight gain, or hypoglycemia, unlike traditional antidiabetic agents [6o]. Their mode of action lies in the effective mediation of glucose reabsorption in the kidney by blocking glucose entry into the renal proximal tubular cell; thereby, causing glucose excretion via urine. Decreased proximal tubular glucose reabsorption with SGLT2 inhibition distributes the burden of salt balance in the early proximal tubule to the distal segments of nephrons. At the same time, increased sodium delivery to the macula densa restores tubuloglomerular feedback, which modulates the tone of glomerular afferent resistance through the release of the vasoconstrictive substance adenosine, which further mitigates glomerular hyperfiltration (Fig. 3) [61] This net reduction in glomerular hyperfiltration helps break the vicious circle that is implicated in the early phase of DN. Moreover, the renoprotective effect of SGLT2 inhibitors, particularly empagliflozin, has been demonstrated through a decrease in high glucose-induced inflammatory and fibrotic markers in human proximal tubular cells. High glucose-induced expression of Toll-like receptor-4, binding of nuclear deoxyribonucleic acid to nuclear factor $\kappa \mathrm{B}$ and activator protein 1 , and secretion of collagen IV and interleukin- 6 were attenuated with empagliflozin; these effects were most likely due to blocking glucose entry into the cell [62].

Several trials have evaluated the potential renoprotective effect of SGLT2 inhibitors in large cohorts of diabetic patients. In one trial, patients treated with canagli- flozin had a decrease in the urinary albumin-creatinine ratio and a slight decrease in eGFR [63]. The slight decrease in eGFR required a dose adjustment in those with renal impairment. On the other hand, this calls into question the reliability of GFR as a trusted variable, especially in those with early phase diabetes with hyperfiltrating nephrons. Moreover, drug dose-dependent hyperkalemia and urogenital infections were reported from time to time with negligible clinical impact [64]. In the most recent trial involving diabetic patients with moderately decreased renal function, the dapagliflozin group showed favorable results in terms of reduced albuminuria [65]. Along with this, favorable metabolic effects, including a reduction in $\mathrm{HbAlc} \mathrm{levels,} \mathrm{decreases} \mathrm{in}$ body weight and blood pressure due to osmotic diuresis, and a loss of calories related to glycosuria, indicate the potential therapeutic effect of SGLT2 inhibition in DN (Fig. 3) $[66,67]$.

\section{5 'Adenosine monophosphate-activated protein kinase activators}

An imbalance between energy intake and expenditure gives rise to alterations in glucose and lipid metabolism that eventually lead to the development of fasting and postprandial hyperglycemia, together with dyslipidemia and insulin resistance, which constitute the characteristic features of diabetes [68]. Chronic exposure to glucose overload, free fatty acids, and amino acids exerts toxic effects in various relevant organs [69]. In this respect, $\mathrm{DN}$ is the end result of multifactorial processes that stem partly from this glucolipotoxicity. A 5' adenosine monophosphate-activated protein kinase (AMPK) is a metabolic master switch that regulates downstream signals based on shifts in surrounding energy reservoirs [70]. There is mounting evidence demonstrating that the dysregulation of AMPK in relevant tissues is implicated in the development of metabolic syndrome and diabetes [70]. As AMPK activation is proven to improve glucose and lipid homeostasis in insulin-resistant animal models by coordinating anabolic processes, targeting this enzyme may ameliorate some of the pathologic features of DN [71,72].

AMPK is activated during metabolic stress in which adenosine triphosphate (ATP) consumption causes an increase in the adenosine monophosphate (AMP)/ATP ratio [7o]. It is also stimulated by several upstream ki- 


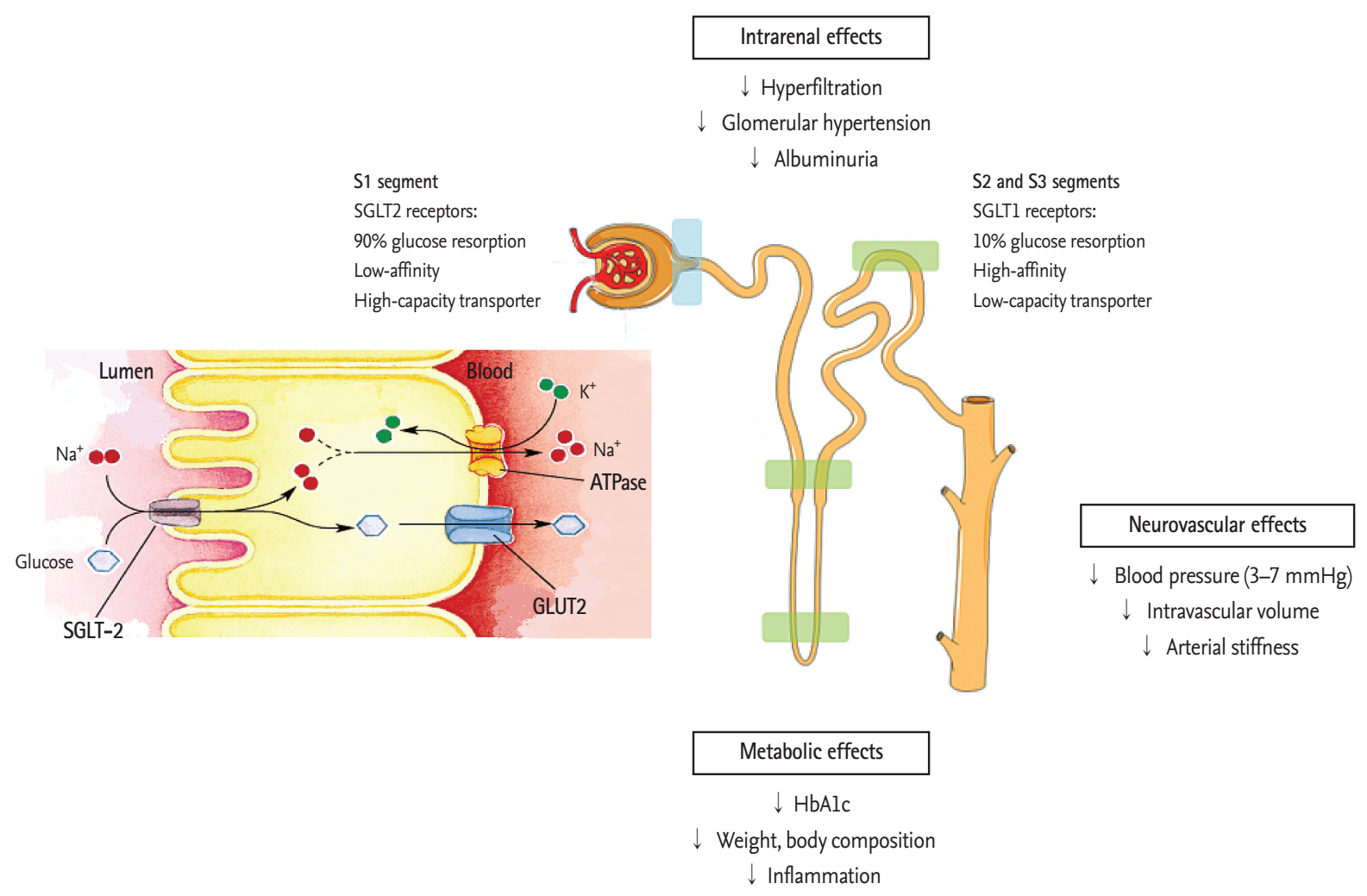

Figure 3. Potential therapeutic effects of sodium/glucose co-transporter 2 (SGLT2) inhibition in diabetic nephropathy. Decreased proximal tubular glucose reabsorption with SGLT2 inhibition results in reduced glomerular hyperfiltration and hypertension with further reduction in albuminuria. Increased sodium delivery to the macula densa modulates tubuloglomerular feedback and contributes to decreased blood pressure, intravascular volume, and arterial stiffness. Metabolic effects of SGLT2 inhibition include reduced glycated hemoglobin (HbA1c), inflammation and weight loss. GLUT2, glucose transporter 2.

nases, including a compound molecule that consists of three proteins: STE-related adaptor (STRAD), mouse protein 25 (MO25), and the tumor-suppressor liver kinase $\mathrm{B} 1$ (LKB1). $\mathrm{Ca}^{2+} /$ calmodulin-dependent protein kinase kinase $\beta$ (CaMKK $\beta$ ) and TGF $\beta$-activated kinase $(\mathrm{TAK} 1)$ are upstream enzymes that participate in the cellular signaling cascade of AMPK activation [73,74]. In response to oxidative stress, activated AMPK phosphorylates its main downstream targets, acetyl-CoA carboxylase and hydroxymethylglutaryl CoA reductase. These are primarily involved in the rate-limiting steps of lipid homeostasis, which further promote fatty acid oxidation upon phosphorylation by AMPK [75]. AMPK activation triggers several adaptive responses for cell survival, which are induced by enhanced oxidative stress due to mitochondrial dysfunction. These downstream targets of AMPK include tuberous sclerosis complex 2, peroxisome proliferator-activated receptor $\gamma$ (PPAR $\gamma)$ coactivator-1 $\alpha$ (PGC-1 $\alpha$ ), and Forkhead box $\mathrm{O}_{3}\left(\mathrm{FOXO}_{3}\right)$, which, upon phosphorylation by AMPK, fortify cellular autophagy, antioxidant defense, and mitochondrial biogenesis through the inhibition of the mammalian target of rapamycin complex 1 in affected cells (Fig. 4) [76]. The sirtuin 1 (Sirtı) protein is the founding member of a family of $\mathrm{NAD}^{+}$-dependent deacetylases and is linked to longevity associated with calorie reduction. Sirtı activity is regulated via the availability of its substrate, $\mathrm{NAD}^{+}$ [77]. In type 2 diabetic animal models $[72,78]$ and human kidneys [79], the expression and activity of Sirtı was significantly reduced. The mechanism of Sirtı reduction in the diabetic condition has not been clarified, but a reduction in the phosphorylation of AMPK may play a role [72]. This notion has been supported by recent publications, which showed that AMPK activation by resveratrol and theobromine leads to Sirtı activation, which protects the diabetic kidney (Fig. 4) [72]. 


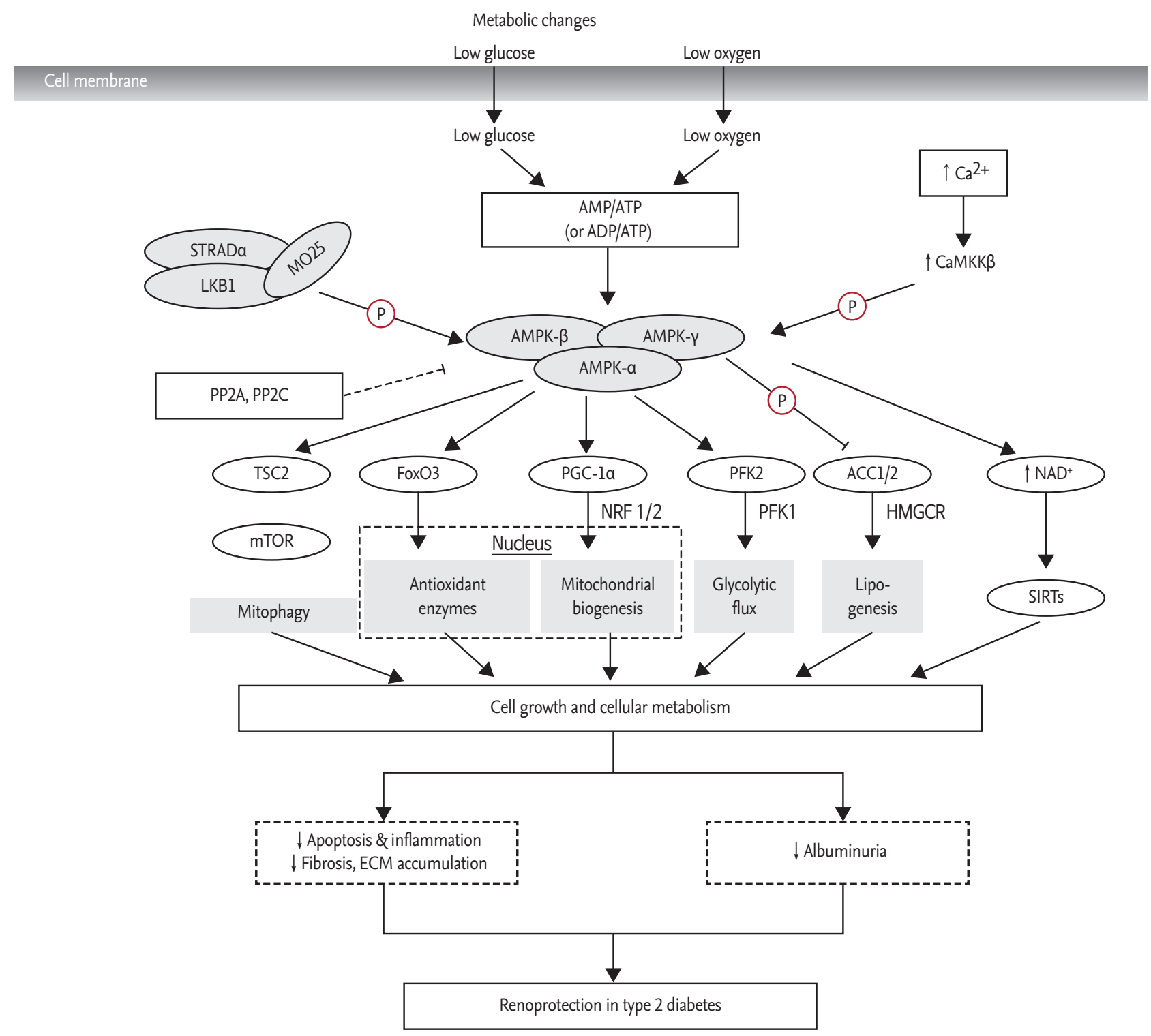

Figure 4. Proposed renoprotective mechanisms in diabetic nephropathy (DN) through adenosine monophosphate-activated protein kinase (AMPK) activation. The AMPK signaling pathway responds to energy stresses that cause fluctuations and imbalances in the adenosine monophosphate/adenosine triphosphate (AMP/ATP) (or adenosine diphosphate [ADP]/ATP) ratio. As the ATP level falls, there is an increase in the AMP level, which triggers the activation of the AMPK pathway. The upstream regulator of AMPK, liver kinase B-1 (LKB1), allows for AMP phosphorylation at a specific site on the $\alpha$ subunit of AMPK. Two other subunits, STE-related adaptor (STRAD) and mouse protein 25 (MO25), form a complex with LKB1 to enhance this process. $\mathrm{Ca}^{++} /$calmodulin-dependent protein kinase kinase $\beta(\mathrm{CaMKK} \beta)$, which is induced by an increase in intracellular $\mathrm{Ca}^{++}$, is also able to activate AMPK. AMPK activation then stimulates its downstream signaling pathways which are involved in oxidative stress, mitochondrial biogenesis, glycolytic flux and lipogenesis that modulate cell growth and metabolism. AMPK also activates sirtuins (SIRTs), especially Sirt-1, which exerts renoprotective effects via improving cellular metabolism. This consequently exerts favorable renoprotective effects by reducing apoptosis, inflammation, glomerulosclerosis and tubulointerstitial fibrosis, and albuminuria in type 2 DN. Adapted from Kim et al. [79]. ACC, acetyl-CoA carboxylase; FoxO3, Forkhead box O3; HMGCR, 3-hydroxy-3-metylglutaryl-CoA reductase; mTOR, mammalian target of rapamycin; NAD, nicotinamide adenine dinucleotide; NRF, nuclear respiratory factor; PGC-1 $\alpha$, PPAR $\gamma$ coactivator $1 \alpha$; PFK2, phosphofructokinase 2; PP2A, protein phosphatase 2A; TSC2, tuberous sclerosis complex 2; ECM, extracellular matrix. 
Conventional activators of AMPK include 5-aminoimidazole-4-carboxamide ribonucleoside, metformin, adiponectin, and resveratrol, which have been shown to provide additional renoprotective effects in addition to their intrinsic activities [72]. Some of the recently recognized AMPK activators are under investigation. Fenofibrate-fed diabetic mice demonstrated both renal functional and phenotypic improvements through reduced lipotoxicity via stimulation of the AMPK-PGC$1 \alpha$-ERR-1 $\alpha$-FOXO3a signaling pathway [77]. Anthocyanin, a flavonoid in the polyphenol class, has also been shown to attenuate lipotoxicity-related apoptosis and oxidative stress in DN through the activation of AMPK and its downstream effectors [78]. Moreover, our unpublished data suggest cinacalcet as a potential therapeutic agent in ameliorating pathologic alterations in DN. Cinacalcet is a calcimimetic that has been shown to increase intracellular $\mathrm{Ca}^{2+}$ and positively modulate the calcium-sensing receptor and subsequently activate the CaMKK $\beta$-LKB1-AMPK signaling pathway in the kidney. AdipoRon, an adiponectin receptor agonist, is an orally active, synthetic molecule that has demonstrated favorable effects in DN through the activation of the AMPK and PPAR $\alpha$ pathways via up-regulation of the adiponectin receptor 1 and 2 in our preliminary data. Taken together, there have been constant attempts to verify the favorable renoprotective effects of AMPK activation in diabetic rodent models that have yet to be translated into human data.

\section{CONCLUSIONS}

In response to the constantly growing burden of diabetes and its associated complications including DN, research into novel therapies to treat DN is expanding. Currently, the management strategy for DN consists mainly of antihypertensive and antiproteinuric measures that depend on strict RAAS inactivation. However, these traditional therapies have been suboptimal and there is a clear, unmet need for treatments that offer effective schemes beyond glucose control. The complexity and heterogeneity of the disease entity, along with the ambiguous renal endpoints, which deter accurate appraisal of new drug potency, are making the situation worse. In fact, albuminuria and eGFR do not appear to be reliable variables that reflect the true renal function of each individual with different stages of DN (Fig. 1). Thus, research into original therapies to treat $\mathrm{DN}$ is focusing on the intrinsic renal pathways that intervene with intracellular signaling of both anti-inflammatory and antifibrotic pathways. Mounting evidence in support of the favorable metabolic effects of these novel agents with respect to the renal aspects of DN supports the likelihood of systemic beneficial effects as well. Thus, when translated into clinical use, these novel agents would also address the comorbid factors associated with diabetes, such as obesity and risk of cardiovascular disease.

\section{Conflict of interest}

No potential conflict of interest relevant to this article was reported.

\section{Acknowledgments}

This study was supported by grants from the Basic Science Research Program through the National Research Foundation of Korea (NRF) funded by the Ministry of Education, Science and Technology (CWP: 2016R1A2B2015878) and the Seoul St. Mary's Hospital R\&D Project from the Catholic University of Korea (CWP: 52015Booo100004). We also thank Professor C.W. Yang for his invaluable support in preparing this manuscript.

\section{REFERENCES}

1. Jha V, Garcia-Garcia G, Iseki K, et al. Chronic kidney disease: global dimension and perspectives. Lancet 2013;382:260-272.

2. Foley RN, Murray AM, Li S, et al. Chronic kidney disease and the risk for cardiovascular disease, renal replacement, and death in the United States Medicare population, 1998 to 1999. J Am Soc Nephrol 2005;16:489-495.

3. Keith DS, Nichols GA, Gullion CM, Brown JB, Smith DH. Longitudinal follow-up and outcomes among a population with chronic kidney disease in a large managed care organization. Arch Intern Med 2004;164:659-663.

4. Kim S, Lim CS, Han DC, et al. The prevalence of chronic kidney disease (CKD) and the associated factors to CKD in urban Korea: a population-based cross-sectional epidemiologic study. J Korean Med Sci 2009;24 Suppl:S11-S21. 
5. Jin DC. Major changes and improvements of dialysis therapy in Korea: review of end-stage renal disease registry. Korean J Intern Med 2015;30:17-22.

6. Park CW. Diabetic kidney disease: from epidemiology to clinical perspectives. Diabetes Metab J 2014;38:252-260.

7. Ruggenenti P, Remuzzi G. Nephropathy of type 1 and type 2 diabetes: diverse pathophysiology, same treatment? Nephrol Dial Transplant 2000;15:1900-1902.

8. Barnes DJ, Pinto JR, Viberti GC. The patient with diabetes mellitus. In: Davison AM, Cameron S, Gunfeld JP, Kerr DN, Ritz E, Winearls CG, eds. Oxford Textbook of Clinical Nephrology. 2nd ed. Oxford: Oxford University Press, 1998:723-775.

9. Vallon V, Thomson SC. Renal function in diabetic disease models: the tubular system in the pathophysiology of the diabetic kidney. Annu Rev Physiol 2012;74:351-375.

10. Saad S, Stevens VA, Wassef L, et al. High glucose transactivates the EGF receptor and up-regulates serum glucocorticoid kinase in the proximal tubule. Kidney Int 2005;68:985-997.

11. Nishikawa T, Edelstein D, Du XL, et al. Normalizing mitochondrial superoxide production blocks three pathways of hyperglycaemic damage. Nature 2000;404:787-790.

12. Navarro-Gonzalez JF, Mora-Fernandez C, Muros de Fuentes M, Garcia-Perez J. Inflammatory molecules and pathways in the pathogenesis of diabetic nephropathy. Nat Rev Nephrol 2011;7:327-340.

13. Fioretto P, Mauer M. Histopathology of diabetic nephropathy. Semin Nephrol 2007;27:195-207.

14. Rutledge JC, Ng KF, Aung HH, Wilson DW. Role of triglyceride-rich lipoproteins in diabetic nephropathy. Nat Rev Nephrol 2010;6:361-370.

15. Cooper ME, Jandeleit-Dahm KA. Lipids and diabetic renal disease. Curr Diab Rep 2005;5:445-448.

16. Bobulescu IA. Renal lipid metabolism and lipotoxicity. Curr Opin Nephrol Hypertens 2010;19:393-402.

17. Jiang T, Wang XX, Scherzer P, et al. Farnesoid X receptor modulates renal lipid metabolism, fibrosis, and diabetic nephropathy. Diabetes 2007;56:2485-2493.

18. Herman-Edelstein M, Scherzer P, Tobar A, Levi M, Gafter U. Altered renal lipid metabolism and renal lipid accumulation in human diabetic nephropathy. J Lipid Res 2014;55:561-572.

19. Klag MJ, Whelton PK, Randall BL, et al. Blood pressure and end-stage renal disease in men. N Engl J Med 1996;334:13-18. 2o. Iseki K, Ikemiya Y, Iseki C, Takishita S. Proteinuria and the risk of developing end-stage renal disease. Kidney Int 2003;63:1468-1474.

21. Locatelli F, Marcelli D, Comelli M, et al. Proteinuria and blood pressure as causal components of progression to end-stage renal failure: Northern Italian Cooperative Study Group. Nephrol Dial Transplant 1996;11:461-467.

22. Iseki K, Kinjo K, Iseki C, Takishita S. Relationship between predicted creatinine clearance and proteinuria and the risk of developing ESRD in Okinawa, Japan. Am J Kidney Dis 2004;44:806-814.

23. Abbate M, Zoja C, Remuzzi G. How does proteinuria cause progressive renal damage? J Am Soc Nephrol 2006;17:2974-2984.

24. Basi S, Fesler P, Mimran A, Lewis JB. Microalbuminuria in type 2 diabetes and hypertension: a marker, treatment target, or innocent bystander? Diabetes Care 2008;31 Suppl 2:S194-S201.

25. Perkins BA, Ficociello LH, Roshan B, Warram JH, Krolewski AS. In patients with type 1 diabetes and new-onset microalbuminuria the development of advanced chronic kidney disease may not require progression to proteinuria. Kidney Int 2010;77:57-64.

26. Gaede P, Vedel P, Larsen N, Jensen GV, Parving HH, Pedersen O. Multifactorial intervention and cardiovascular disease in patients with type 2 diabetes. $\mathrm{N}$ Engl J Med 2003;348:383-393.

27. Brenner BM, Cooper ME, de Zeeuw D, et al. Effects of losartan on renal and cardiovascular outcomes in patients with type 2 diabetes and nephropathy. N Engl J Med 2001;345:861-869.

28. Lewis EJ, Hunsicker LG, Clarke WR, et al. Renoprotective effect of the angiotensin-receptor antagonist irbesartan in patients with nephropathy due to type 2 diabetes. $\mathrm{N}$ Engl J Med 2001;345:851-860.

29. Azizi M, Menard J. Combined blockade of the renin-angiotensin system with angiotensin-converting enzyme inhibitors and angiotensin II type 1 receptor antagonists. Circulation 2004;109:2492-2499.

30. Azizi M, Webb R, Nussberger J, Hollenberg NK. Renin inhibition with aliskiren: where are we now, and where are we going? J Hypertens 2006;24:243-256.

31. Muller DN, Luft FC. Direct renin inhibition with aliskiren in hypertension and target organ damage. Clin J Am Soc Nephrol 2006;1:221-228.

32. Persson F, Lewis JB, Lewis EJ, et al. Impact of baseline re- 
nal function on the efficacy and safety of aliskiren added to losartan in patients with type 2 diabetes and nephropathy. Diabetes Care 2010;33:2304-2309.

33. Navaneethan SD, Nigwekar SU, Sehgal AR, Strippoli GF. Aldosterone antagonists for preventing the progression of chronic kidney disease: a systematic review and meta-analysis. Clin J Am Soc Nephrol 2009;4:542-551.

34. Palmer SC, Craig JC, Navaneethan SD, Tonelli M, Pellegrini F, Strippoli GF. Benefits and harms of statin therapy for persons with chronic kidney disease: a systematic review and meta-analysis. Ann Intern Med 2012;157:263-275.

35. Rodrigues CJ. HMG CoA reductase inhibitors (statins) for people with chronic kidney disease not requiring dialysis. Sao Paulo Med J 2015;133:541-542.

36. Mann JF, Green D, Jamerson K, et al. Avosentan for overt diabetic nephropathy. J Am Soc Nephrol 2010;21:527-535.

37. Schievink B, de Zeeuw D, Smink PA, et al. Prediction of the effect of atrasentan on renal and heart failure outcomes based on short-term changes in multiple risk markers. Eur J Prev Cardiol 2016;23:758-768.

38. de Zeeuw D, Akizawa T, Audhya P, et al. Bardoxolone methyl in type 2 diabetes and stage 4 chronic kidney disease. N Engl J Med 2013;369:2492-2503.

39. Packham DK, Wolfe R, Reutens AT, et al. Sulodexide fails to demonstrate renoprotection in overt type 2 diabetic nephropathy. J Am Soc Nephrol 2012;23:123-130.

40. Coyne DW, Andress DL, Amdahl MJ, Ritz E, de Zeeuw D. Effects of paricalcitol on calcium and phosphate metabolism and markers of bone health in patients with diabetic nephropathy: results of the VITAL study. Nephrol Dial Transplant 2013;28:2260-2268.

41. Lindhardt M, Persson F, Currie G, et al. Proteomic prediction and renin angiotensin aldosterone system Inhibition prevention of early diabetic nephropathy in type 2 diabetic patients with normoalbuminuria (PRIORITY): essential study design and rationale of a randomised clinical multicentre trial. BMJ Open 2016;6:e010310.

42. Sircar D, Chatterjee S, Waikhom R, et al. Efficacy of febuxostat for slowing the GFR decline in patients with CKD and asymptomatic hyperuricemia: a 6-month, double-blind, randomized, placebo-controlled trial. Am J Kidney Dis 2015;66:945-950.

43. Navarro-Gonzalez JF, Mora-Fernandez C, Muros de Fuentes $\mathrm{M}$, et al. Effect of pentoxifylline on renal function and urinary albumin excretion in patients with diabetic kidney disease: the PREDIAN trial. J Am Soc Nephrol
2015;26:220-229.

44. Abdel Aziz MT, Wassef MA, Ahmed HH, et al. The role of bone marrow derived-mesenchymal stem cells in attenuation of kidney function in rats with diabetic nephropathy. Diabetol Metab Syndr 2014;6:34.

45. Ezquer F, Giraud-Billoud M, Carpio D, Cabezas F, Conget P, Ezquer M. Proregenerative microenvironment triggered by donor mesenchymal stem cells preserves renal function and structure in mice with severe diabetes mellitus. Biomed Res Int 2015;2015:164703.

46. Vilsboll T, Krarup T, Deacon CF, Madsbad S, Holst JJ. Reduced postprandial concentrations of intact biologically active glucagon-like peptide 1 in type 2 diabetic patients. Diabetes 2001;50:609-613.

47. Ahren B, Larsson H, Holst JJ. Reduced gastric inhibitory polypeptide but normal glucagon-like peptide 1 response to oral glucose in postmenopausal women with impaired glucose tolerance. Eur J Endocrinol 1997;137:127-131.

48. Vilsboll T, Krarup T, Madsbad S, Holst JJ. Defective amplification of the late phase insulin response to glucose by GIP in obese type II diabetic patients. Diabetologia 2002;45:1111-1119.

49. Drucker DJ. Biological actions and therapeutic potential of the glucagon-like peptides. Gastroenterology 2002;122:531-544.

50. Nauck MA, Niedereichholz U, Ettler R, et al. Glucagon-like peptide 1 inhibition of gastric emptying outweighs its insulinotropic effects in healthy humans. Am J Physiol 1997;273(5 Pt 1):E981-E988.

51. Verdich C, Flint A, Gutzwiller JP, et al. A meta-analysis of the effect of glucagon-like peptide-1 (7-36) amide on ad libitum energy intake in humans. J Clin Endocrinol Metab 2001;86:4382-4389.

52. Muskiet MH, Smits MM, Morsink LM, Diamant M. The gut-renal axis: do incretin-based agents confer renoprotection in diabetes? Nat Rev Nephrol 2014;10:88-103.

53. Hattori S. Sitagliptin reduces albuminuria in patients with type 2 diabetes. Endocr J 2011;58:69-73.

54. Sakata K, Hayakawa M, Yano Y, et al. Efficacy of alogliptin, a dipeptidyl peptidase-4 inhibitor, on glucose parameters, the activity of the advanced glycation end product (AGE): receptor for AGE (RAGE) axis and albuminuria in Japanese type 2 diabetes. Diabetes Metab Res Rev 2013;29:624630.

55. Groop PH, Cooper ME, Perkovic V, Emser A, Woerle HJ, von Eynatten M. Linagliptin lowers albuminuria on top 
of recommended standard treatment in patients with type 2 diabetes and renal dysfunction. Diabetes Care 2013;36:3460-3468.

56. Zhang $\mathrm{H}$, Zhang X, Hu C, Lu W. Exenatide reduces urinary transforming growth factor-betar and type IV collagen excretion in patients with type 2 diabetes and microalbuminuria. Kidney Blood Press Res 2012;35:483-488.

57. Kanasaki K, Shi S, Kanasaki M, et al. Linagliptin-mediated DPP-4 inhibition ameliorates kidney fibrosis in streptozotocin-induced diabetic mice by inhibiting endothelial-to-mesenchymal transition in a therapeutic regimen. Diabetes 2014;63:2120-2131.

58. Wong MG, Panchapakesan U, Qi W, Silva DG, Chen XM, Pollock CA. Cation-independent mannose 6-phosphate receptor inhibitor ( $\mathrm{PXS}_{25}$ ) inhibits fibrosis in human proximal tubular cells by inhibiting conversion of latent to active TGF-betal. Am J Physiol Renal Physiol 2011;301:F84-F93.

59. Panchapakesan U, Pollock CA. DPP-4 inhibitors-renoprotection in diabetic nephropathy? Diabetes 2014;63:18291830 .

6o. Ghosh RK, Ghosh SM, Chawla S, Jasdanwala SA. SGLT2 inhibitors: a new emerging therapeutic class in the treatment of type 2 diabetes mellitus. J Clin Pharmacol 2012;52:457-463.

61. Thomson SC, Rieg T, Miracle C, et al. Acute and chronic effects of SGLT2 blockade on glomerular and tubular function in the early diabetic rat. Am J Physiol Regul Integr Comp Physiol 2012;302:R75-R83.

62. Panchapakesan U, Pegg K, Gross S, et al. Effects of SGLT2 inhibition in human kidney proximal tubular cells: renoprotection in diabetic nephropathy? PLoS One 2013;8:e54442.

63. Cefalu WT, Leiter LA, Yoon KH, et al. Efficacy and safety of canagliflozin versus glimepiride in patients with type 2 diabetes inadequately controlled with metformin (CANTATA-SU): 52 week results from a randomised, double-blind, phase 3 non-inferiority trial. Lancet 2013;382:941-950.

64. Nicolle LE, Capuano G, Ways K, Usiskin K. Effect of canagliflozin, a sodium glucose co-transporter 2 (SGLT2) inhibitor, on bacteriuria and urinary tract infection in subjects with type 2 diabetes enrolled in a 12-week, phase 2 study. Curr Med Res Opin 2012;28:1167-1171.

65. Kohan DE, Fioretto P, Tang W, List JF. Long-term study of patients with type 2 diabetes and moderate renal impairment shows that dapagliflozin reduces weight and blood pressure but does not improve glycemic control. Kidney Int 2014;85:962-971.

66. Musso G, Gambino R, Cassader M, Pagano G. A novel approach to control hyperglycemia in type 2 diabetes: sodium glucose co-transport (SGLT) inhibitors: systematic review and meta-analysis of randomized trials. Ann Med 2012;44:375-393.

67. Vasilakou D, Karagiannis T, Athanasiadou E, et al. Sodium-glucose cotransporter 2 inhibitors for type 2 diabetes: a systematic review and meta-analysis. Ann Intern Med 2013;159:262-274.

68. Viollet B, Lantier L, Devin-Leclerc J, et al. Targeting the AMPK pathway for the treatment of type 2 diabetes. Front Biosci (Landmark Ed) 2009;14:3380-3400.

69. Dronavalli S, Duka I, Bakris GL. The pathogenesis of diabetic nephropathy. Nat Clin Pract Endocrinol Metab 2008;4:444-452.

70. Hardie DG, Ross FA, Hawley SA. AMPK: a nutrient and energy sensor that maintains energy homeostasis. Nat Rev Mol Cell Biol 2012;13:251-262.

71. Hallows KR, Mount PF, Pastor-Soler NM, Power DA. Role of the energy sensor AMP-activated protein kinase in renal physiology and disease. Am J Physiol Renal Physiol 2010;298:F1067-F1077.

72. Kim MY, Lim JH, Youn HH, et al. Resveratrol prevents renal lipotoxicity and inhibits mesangial cell glucotoxicity in a manner dependent on the AMPK-SIRT1-PGC1alpha axis in $\mathrm{db} / \mathrm{db}$ mice. Diabetologia 2013;56:204-217.

73. Hawley SA, Selbert MA, Goldstein EG, Edelman AM, Carling D, Hardie DG. 5'-AMP activates the AMP-activated protein kinase cascade, and $\mathrm{Ca2}+$ /calmodulin activates the calmodulin-dependent protein kinase I cascade, via three independent mechanisms. J Biol Chem 1995;270:27186-27191.

74. Momcilovic M, Hong SP, Carlson M. Mammalian TAKı activates Snfi protein kinase in yeast and phosphorylates AMP-activated protein kinase in vitro. J Biol Chem 2006;281:25336-25343.

75. Hawley SA, Boudeau J, Reid JL, et al. Complexes between the LKB1 tumor suppressor, STRAD alpha/beta and MO25 alpha/beta are upstream kinases in the AMP-activated protein kinase cascade. J Biol 2003;2:28.

76. Sanders MJ, Ali ZS, Hegarty BD, Heath R, Snowden MA, Carling D. Defining the mechanism of activation of AMP-activated protein kinase by the small molecule A-769662, a member of the thienopyridone family. J Biol 
Chem 2007;282:32539-32548.

77. Hong YA, Lim JH, Kim MY, et al. Fenofibrate improves renal lipotoxicity through activation of AMPK-PGC-1alpha in $\mathrm{db} / \mathrm{db}$ mice. PLoS One 2014;9:e96147.

78. Koh ES, Lim JH, Kim MY, et al. Anthocyanin-rich Seoritae extract ameliorates renal lipotoxicity via activation of
AMP-activated protein kinase in diabetic mice. J Transl Med 2015;13:203.

79. Kim Y, Park CW. Adenosine monophosphate-activated protein kinase in diabetic nephropathy. Kidney Res Clin Pract 2016;35:69-77. 